\title{
The role of human papilloma virus in oral squamous cell carcinoma
}

\begin{abstract}
Oral squamous cell carcinoma (OSCC) that is estimated to have more than $90 \%$ of all oral neoplasms is the most frequently seen malignancy affecting the oral cavity. Besides the tobacco, alcohol, genetic predisposition and hormonal factors, viruses such as human papilloma viruses (HPV) play an important role in the etiology of SCC of head and neck region. The increasing number of OSCC cases and high probability of correlation with OSCC and viral factors, including HPV alert to bring light to the issue. The aim of this review is to highlight the current understanding of HPVassociated oral cancer with an emphasis on its prognosis, detection and management.
\end{abstract}

Keywords: hpv, squamous cell carcinoma, oral cancer
Volume 4 Issue 4 - 2017

\author{
Dilek Aynur Ugar Cankal, Suleyman Bozkaya \\ Department of Oral and Maxillofacial, Gazi University, Turkey
}

Correspondence: Dilek Aynur Ugar Cankal, Department

of Oral and Maxillofacial, Gazi University, Turkey, Tel

+905323482295, Fax +903122239226,

Email dilekaynur@gmail.com

Received: August 01, 2017| Published: August 28, 2017
Abbreviations: OSCC, oral squamous cell carcinoma; HPV, human papilloma viruses; HNC, head and neck cancer; SCC, squamous cell carcinoma; $\mathrm{RB}$, retino blastoma

\section{Introduction}

Head and neck cancer (HNC) is a broad term used to describe a group of neoplasms affecting the oral cavity, or pharynx, hypo pharynx and the larynx. It is estimated that more than $90 \%$ of these malignancies are squamous cell carcinoma (SCC). ${ }^{1,2} \mathrm{HNC}$ causes significant morbidity and mortality, especially in low socioeconomic groups. ${ }^{3}$ Studies have estimated the global incidence of all HNCs to be between 400,000 and 600,000 new cases per year and the mortality rate to between 223,000 and 300,000 deaths per year, ${ }^{4}$ and its incidence is higher in Melanesian and South Central Asia countries and in Central and Eastern Europe., 3,52016 data revealed 48,330 newly diagnosed cases with SCCs and 9,570 expected related deaths in the USA alone. ${ }^{7}$ In Europe, the situation is not different. About 99,630 new cases of HNC were reported in 2012, and 43,704 cases of deaths were reported in the same year. ${ }^{2}$ The five-year overall survival rate of patients with head and neck squamous cell carcinoma is about $40-50 \%{ }^{4}$

Head and neck squamous cell carcinoma (HNSCC) are typically regarded as diseases of the elderly and predominantly seen in men in their sixth and seventh decades. ${ }^{1,2,6}$ However, last 3 decades, increasing numbers of patients worldwide are being diagnosed with head and neck SCC at a younger age ( $<45$ years old). ${ }^{2}$ It is noteworthy that the demographic pattern of this disease among young patients is different with regard to the aetiological factors and gender. Whereas human papillomavirus (HPV)-related oropharyngeal cancers are more likely to occur in men who are non-smokers, non-drinkers and have a good socio-economic status, oral (freely mobile portion) tongue cancer mostly affects young white women with unknown causes at the moment. ${ }^{2,4} \mathrm{SCC}$ in head and neck region has been connected with tobacco and tobacco-related products as betel quid, smokeless tobacco, and alcohol, genetic predisposition and hormonal factors. ${ }^{1,3,8}$ During the past few decades, several countries have observed a decline in oral cavity alcohol exposure cancer incidence correlating to a decline in tobacco use. However, Denmark, Norway, Sweden, Canada, the Netherlands, the US, and the UK, have seen an increasing rate of oropharyngeal and oral cavity cancers despite declines in smoking rates since the $1980 \mathrm{~s} .{ }^{4,8}$ This has led to theories that human papillomavirus (HPV) infection might be an additional risk factor for developing certain head and neck cancers. Recent studies also report that viral factors, including human papilloma viruses (HPV), may contribute to the aetiology of this malignant neoplasm. ${ }^{1,3,8}$

HPV is a complex group of viruses that include more than 150 known strains and small, epitheliotropic, no enveloped, doublestranded DNA viruses that belong to the family papilloma viridae. ${ }^{1,9}$ HPV infections are transmitted by direct skin-to-skin contact. HPV is a sexually transmitted disease and is common among young adults. Because the penetrative sex is the most frequent route of transmission and young adults are tend to engage in risky sexual behaviors, ${ }^{9}$ they are more likely to be considered in high- risk group 9. Some HPV types because warts on the skin so are called "cutaneous". HPVs are also called papilloma viruses because some of the HPV types such as HPV-6 and HPV-11 cause benign oral lesions. However, some types of HPVs such as HPV-31, HPV-33 and HPV-35 are HPV subtypes that have high oncogenic risk. HPV-1, HPV-2, HPV-4, HPV-7, HPV57 cause potentially malignant oral lesions and HPV-16 and HPV18 have been highly recognized to cause oral and oropharyngeal squamous cell carcinoma1. Recently, HPV type 16 has been identified in $90 \%$ of HPV-associated head and neck tumors and has been found in $50 \%$ of oropharyngeal head and neck squamous cell carcinomas. ${ }^{1,8}$ HPV prevalence is notably high in oropharyngeal cancer, in which HPV16 is present at high copy number, localized to cell nuclei, frequently integrated into the cell genome, and actively transcribing viral oncoproteins E6 and E7. ${ }^{10}$

Transformation of oral epithelial cells can be brought about by high risk oncogenic subtypes HPV-16, HPV-18, HPV-31, HPV- 33 and HPV-351. The infection caused by HPV in humans leads latent infection. Due to this infection, E6 and E7 proteins are formed in the host genome. These proteins alter tumour suppressor pathways by inactivating $\mathrm{p} 53$ and the retinoblastoma gene $(\mathrm{Rb}) 1$ E6 binds to a cellular ubiquitin/protein ligase and to the tumour suppressor protein p53. E6 oncoproteins degrade the p53 and increase the cell proliferation in HPV-associated carcinomas. It is pointed out that p53 polymorphism (Arg72Pro) increases the odds of oral cancer in the presence of HPV infection. ${ }^{3}$ The HPV-positive OSCC appears to be different from HPV-negative SCC, both in its molecular and 
clinical features. ${ }^{1}$ The HPV -positive SCC is characterized by genetic alterations, clinical progression, and therapeutic response. Patients with HPV-positive oropharyngeal squamous cell carcinoma have significantly better survival. PCR or in situ hybridization is the techniques for the detecting HPV in OSCC. The histopathological diagnosis of HPV infection is based on the presence of kilobytes, dyskeratosis, papillomatosis, hyperkeratosis, acanthuses and parakeratosis. Progression of cancer can be determined by using novel biomarkers. ${ }^{1}$ HPV vaccines to prevent genital HPV infection are licensed in many countries worldwide. ${ }^{8}$ Although the use of HPV vaccines to prevent $\mathrm{HPV}$-positive OSCC is not yet recommended by the World Health Organization (WHO), the development of HPV vaccine offers hope for prevention of cancers of the or pharynx and oral cavity. Oral HPV prevalence, four years subsequent to HPV 16/18 vaccination, revealed strong protection against oral HPV $16 / 18$ infection, suggesting that the vaccine can provide strong protection against HPV-associated oropharyngeal cancer.11 Patients with HPV-positive oropharyngeal squamous cell carcinoma have a better prognosis and respond more favorably to chemotherapy and/or radiotherapy than do patients with HPV-negative SCC. Therefore, it is important to comprehend how altered molecular pathways in HPVpositive HNSCC influence the behavior and response of CSCs to the tumor microenvironment. This will provide basis to the treatment of oropharyngeal squamous cell carcinoma. ${ }^{8}$

\section{Conclusion}

Survival differences by HPV DNA presence have been consistently reported exclusively for oropharyngeal cancers. These features support, for the moment, a causal role of HPV infection in some oropharyngeal cancers, whose incidence is currently increasing in some developed countries. Further researches are needed to clarify the details of HPV related OSCC.

\section{Acknowledgements}

We thank to the editors of the journal for the invitation to write this manuscript.

\section{Conflict of interest}

We have not any financial interest or any conflict of interest.

\section{References}

1. Khot KP, Deshmane S, Choudhari S. Human Papilloma Virus in Oral Squamous Cell Carcinoma-The Enigma Unravelled. Chin J Dent Res. 2016;19(1):17-23.

2. Hussein AA, Helder MN, de Visscher JG, et al. Global incidence of oral and oropharynx cancer in patients younger than 45 years versus older patients:A systematic review. Eur J Cancer. 2017;82:115-127.

3. Ali J, Sabiha B, Jan HU, et al. Genetic etiology of oral cancer. Oral Oncol. 2017;70:23-28.

4. Head And Neck Cancer Union for International Cancer Control. USA: Review of Cancer Medicines on the WHO List of Essential Medicines; 2014. p. $1-8$.

5. Saba NF, Goodman M, Ward K, et al. Gender and ethnic disparities in incidence and survival of squamous cell carcinoma of the oral tongue, base of tongue, and tonsils: a surveillance, epidemiology and end results program-based analysis. Oncology. 2011;81(1):12-20.

6. Ferlay J, Soerjomataram I, Dikshit R, et al. Cancer incidence and mortality worldwide: sources, methods and major patterns in GLOBOCAN 2012 Int J Cancer. 2015;136(5):E359-E386.

7. Siegel RL, Miller KD, Jemal A. Cancer statistics, 2016. A Cancer J Clin. 2016;66(1):7-30.

8. Pullos AN, Castilho RM, Squarize CH. HPV Infection of the Head and Neck Region and Its Stem Cells. Dent Res. 2015;94(11):1532-1543.

9. Minassian M. HPV-positive head and neck cancers: a review of the literature. J Dent Hyg. 2014;88(4):194-201.

10. Bernard W Stewart, Christopher P Wild. World Cancer Report 2014. Switzerland: World Health Organization, International Agency for Research on Cancer, WHO Press; 2015.

11. Herrero R, Quint W, Hildesheim A, et al. Reduced prevalence of oral human papillomavirus (HPV) 4 years after bivalent $\mathrm{HPV}$ vaccination in a randomized clinical trial in Costa Rica. PLoS One. 2013;8(7):e68329. 